\title{
The Association and Mechanism between Obstructive Sleep Apnea and Systemic/Pulmonary Hypertension
}

\author{
Jin Oh Na'1, Woohyeun Kim², Dong Oh Kang' ${ }^{1}$, and Jah Yeon Choi ${ }^{1}$ \\ ${ }^{1}$ Cardiovascular Center, Korea University Guro Hospital, Seoul, Korea \\ ${ }^{2}$ Division of Cardiology, Department of Internal Medicine, Hanyang University College of Medicine, Seoul, Korea
}

\begin{abstract}
Sleep apnea and hypertension are known to be closely related to the development of the cardiovascular disease, so these diseases must be treated properly. Moreover, it has been continuously reported that sleep apnea syndrome and hypertension are highly correlated, and they share not only many risk factors such as obesity, but also some pathophysiological mechanisms. The ultimate aim of treatment for hypertension is not simply to lower blood pressure but to prevent cardiovascular diseases such as angina, myocardial infarction, stroke, heart failure, and death. Sleep apnea treatment is similarly aimed at preventing related complications and lowering mortality by controlling many risk factors including the occurrence of hypertension. It is important to understand the pathophysiology of the relationship between sleep apnea and hypertension. Thereby we can understand why physicians should pay more attention and why they should treat sleep apnea.
\end{abstract}

Key Words: Sleep apnea; Hypertension; Pathophysiology

Received: October 26, 2020 Revised: November 29, 2020 Accepted: November 30, 2020

Corresponding author: Jin Oh Na, MD, PhD, Cardiovascular Center, Korea University Guro Hospital, 148 Gurodong-ro, Guro-gu, Seoul 08308, Korea.

Tel: 82-2-2626-3025, Fax: 82-2-863-1109, E-mail: koolup93@gmail.com

@ This is an Open Access article distributed under the terms of the Creative Commons Attribution Non-Commercial License (https://creativecommons.org/licenses/by$\mathrm{nc/4.0)}$ which permits unrestricted non-commercial use, distribution, and reproduction in any medium, provided the original work is properly cited.

\section{INTRODUCTION}

Both sleep apnea syndrome and hypertension are subjects that have been studied for a long time. Sleep apnea and hypertension are known to be closely related to the development of cardiovascular disease, so these diseases must be treated properly [1-3]. Moreover, it has been continuously reported that these sleep apnea syndrome and hypertension are highly correlated, and they share not only many risk factors such as obesity, but also some pathophysiological mechanisms [4]. However, since many other factors are also involved between these two diseases, it is difficult to say that there is a strong causal relationship between the two diseases, but it is clear that the relationship cannot be ignored.

As the ultimate treatment for hypertension is not simply to lower blood pressure, but to prevent cardiovascular diseases such as angina, myocardial infarction, stroke, heart failure, arrhythmia, and death, sleep apnea treatment aims to prevent lower complications and mortality by controlling many risk factors including the occurrence of hypertension [2,5-7].
There has also been reported a link between sleep apnea syndrome and pulmonary hypertension. Sleep apnea syndrome can cause or worsen pulmonary hypertension [8]. Therefore, if the cause of pulmonary hypertension is not clear, polysomnography can be performed to confirm sleep apnea syndrome, and it has been reported that continuous positive airway pressure (CPAP) treatment is useful for lowering pulmonary arterial pressure in the patients with sleep apnea $[9,10]$.

In this brief review, the relationship between sleep apnea syndrome and hypertension is discussed including mechanisms and recent important studies.

\section{PATHOPHYSIOLOGIC MECHANISMS BETWEEN SLEEP APNEA SYNDROME AND HYPERTENSION}

When obstructive sleep apnea occurs due to upper airway obstruction during sleep, the sympathetic nervous system is activated and the level of serum catecholamine and blood pressure 
growing increasing [11]. At this time, the heart rate changes according to the changes in the parasympathetic and sympathetic nerves, which may rise or fall. When apnea is started, blood pressure tends to decrease slightly at first. But, while apnea continues, blood oxygen saturation $\left(\mathrm{SaO}_{2}\right)$ gradually decreases, and blood pressure and heart rate gradually increases due to activation of the sympathetic nervous system. When apnea is over and the moment start breathing again, heart rate and blood pressure reach the peak, while the blood oxygen concentration becomes the lowest. At the same time, activation of the sympathetic nervous system and arousal during sleep appear repeatedly, and this pattern continues to repeat during whole sleep period (Figure 1) [12]. In addition, as $\mathrm{SaO}_{2}$ decreases, vasoconstriction of peripheral artery and pulmonary artery results increasing peripheral and pulmonary vascular resistance. As a result, it causes a reduction in left ventricular preload, resulting in a drop in cardiac output that is temporary but repeats throughout sleep [13]. When the blood pressure rises due to sleep apnea, it affects the baroreceptor of the aortic arch and carotid artery and activates the parasympathetic nerve, which causes the blood pressure to fall again and the respiratory rate to increase. It is believed that continuous exposure to sleep apnea leads to a weakening of the compensation mechanism, making it difficult to properly control blood pressure [11].

Therefore, patients with sleep apnea tend to have high overall blood pressure during sleep, which is expressed by non-dipper patients who do not show a physiological 10-20\% decrease in blood pressure during sleep. Sleep apnea should be suspected in these non-dipper patients $[4,14]$.

\section{SYSTEMIC HYPERTENSION AND SLEEP APNEA}

Hypertension is a representative controllable risk factor for cardiovascular disease, and according to the National Health and $\mathrm{Nu}-$ trition Survey, the prevalence of hypertension in adults over the age of 30 in Korea is reported as $29.1 \%$ [15]. Sleep apnea is diagnosed when the apnea-hypopnea index is more than 5 times per hour. In Korea, the prevalence rate is about $27 \%$ for men and $16 \%$ for women [16]. It has been continuously reported that hypertension and sleep apnea are closely related. According to actual epidemiological data, the prevalence of sleep apnea in hypertensive patients reaches $30-50 \%$, and conversely, the prevalence of hypertension in sleep apnea patients reaches $50 \%[17,18]$. It can be inferred that the two diseases are closely associated because the prevalence of the two diseases is higher when combined than the prevalence of each single disease.

In a prospective cohort study conducted in Spain, 1889 patients with sleep apnea without hypertension were followed up for 12.2 years, and the incidence of hypertension was higher in patients with sleep apnea. The more severe the degree of sleep apnea, the higher the incidence of hypertension (Figure 2) and the incidence of hypertension is significantly lowered when CPAP therapy is

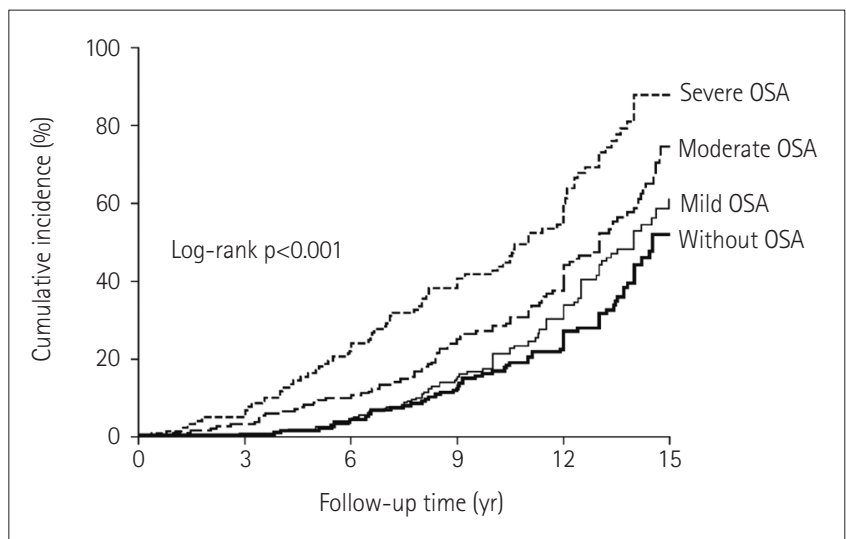

Figure 2. The cumulative incidence of hypertension in people who do not have sleep apnea and who have not been treated for sleep apnea. It can be seen that the more severe the degree of sleep apnea, the higher the incidence of hypertension. OSA: obstructive sleep apnea. Adapted from Marin et al. JAMA 2012;307:2169-2176, with permission of American Medical Association [19].

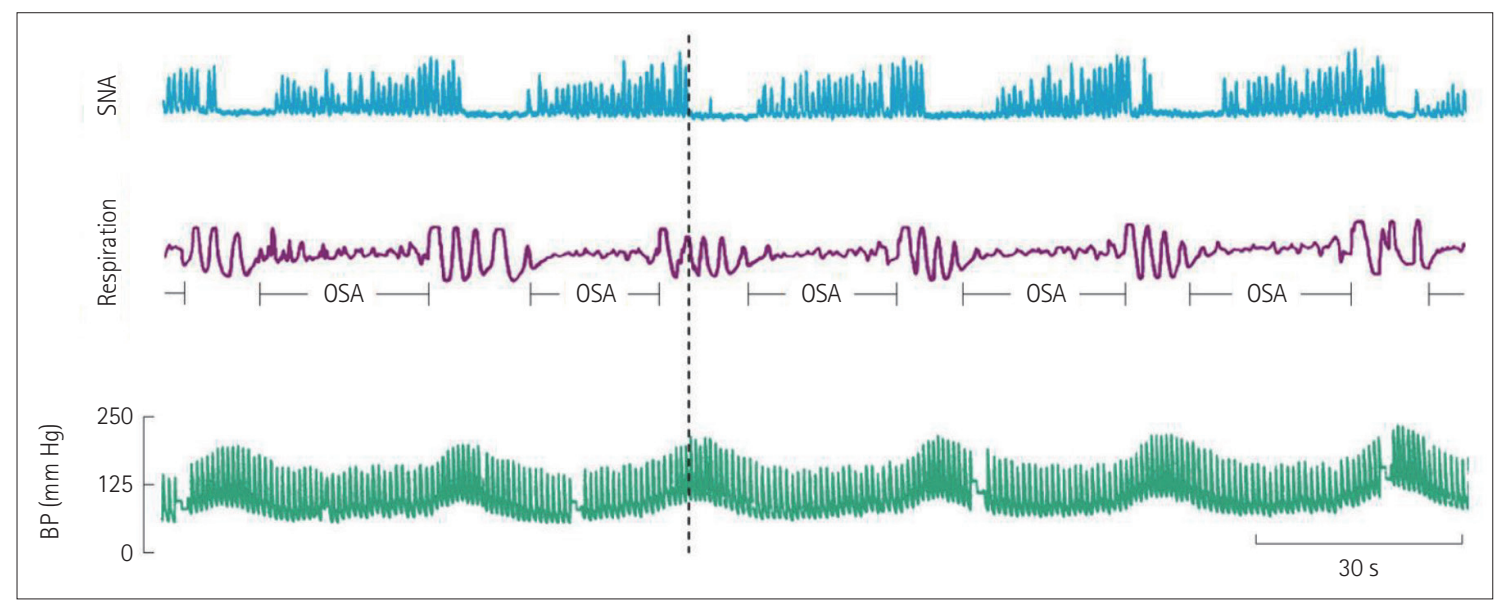

Figure 1. The pattern of continuous and repetitive changes in the sympathetic nervous system, respiration, and BP that occur as a reaction to repeated sleep apnea. As sleep apnea occurs, sympathetic nervous system activation occurs, followed by BP rising. This change occurs throughout sleep, and the vertical dotted line shows that the peak in BP and activation of the sympathetic nervous system suddenly drop as soon as the respiratory system resumes. SNA: sympathetic nerve activity, OSA: obstructive sleep apnea, BP: blood pressure. Adapted from Somers et al. J Clin Invest 1995;96:1897-1904, with permission of American Society for Clinical Investigation [12]. 
used effectively for more than 4 hours every day [19,20].

As such, hypertension and sleep apnea are closely related bidirectional pattern. In hypertensive patients, sleep apnea is likely to be accompanied, and conversely, in sleep apnea patients, hypertension is also more likely to be occurred. In particular, as sleep apnea is more severe, hypertension is more likely to have occurred. Thus, it is believed that the occurrence of hypertension can be reduced if appropriate treatment is accompanied. In conclusion, sleep apnea is known as an independent risk factor for causing hypertension, and in addition, it is recognized as an independent risk factor for pulmonary hypertension, stroke, coronary artery disease, arrhythmia, and heart failure.

\section{RESISTANT HYPERTENSION AND SLEEP APNEA}

It is known that the prevalence of resistant hypertension accompanied by sleep apnea is much higher than that of general hypertensive patients. The reason is that the pathophysiological mechanism of resistant hypertension is usually understood as a result of changes in the renin-angiotensin-aldosterone axis or excessive activation of the sympathetic nervous system, as sleep apnea patients also share this mechanism. In other words, sleep apnea is directly involved in the same mechanism of resistant hypertension [14]. In fact, a comprehensive epidemiologic study shows that sleep apnea is accompanied by about $30 \%$ to $50 \%$ of patients with simple hypertension [18], whereas it is accompanied by a very high rate of about $70 \%$ to $85 \%$ of patients with resistant hypertension $[21,22]$. There are even studies that report over $90 \%$ [23]. In addition, sleep apnea is the most common cause of resistance to drug treatment for hypertension, accounting for more than half [24]. These patients take more drugs to control blood pressure, and complications such as diabetes, cardiovascular disease, stroke, and chronic kidney failure are known to be more prone. Therefore, it is necessary to investigate whether there is sleep apnea, one of the biggest causes. Moreover, even if there is sleep apnea in these resistant hypertensive patients, it is very common that there are few subjective symptoms such as daytime sleepiness, so do not rely only on the patient's statement. A sleep apnea should be actively questioned through a sleep partner or a sleep-related questionnaire, and a polysomnography should be performed if necessary.

In patients with resistant hypertension, when sleep apnea is accompanied, continuous positive pressure therapy is known to be more effective than in patients with simple hypertension. When CPAP therapy in patient with simple hypertension was performed, systolic and diastolic blood pressure decreased by about 2.6/2.0 $\mathrm{mm} \mathrm{Hg}$, whereas in patients with resistant hypertension, 7.2/5.0 $\mathrm{mm} \mathrm{Hg}$ fell, respectively [25]. Therefore, more blood pressure lowering effects can be expected when sleep apnea treatment is performed in patients with resistant hypertension (Figure 3) [14]. This is thought to be due to the fact that proper sleep apnea treatment reduces the level of aldosterone in addition to the effect of

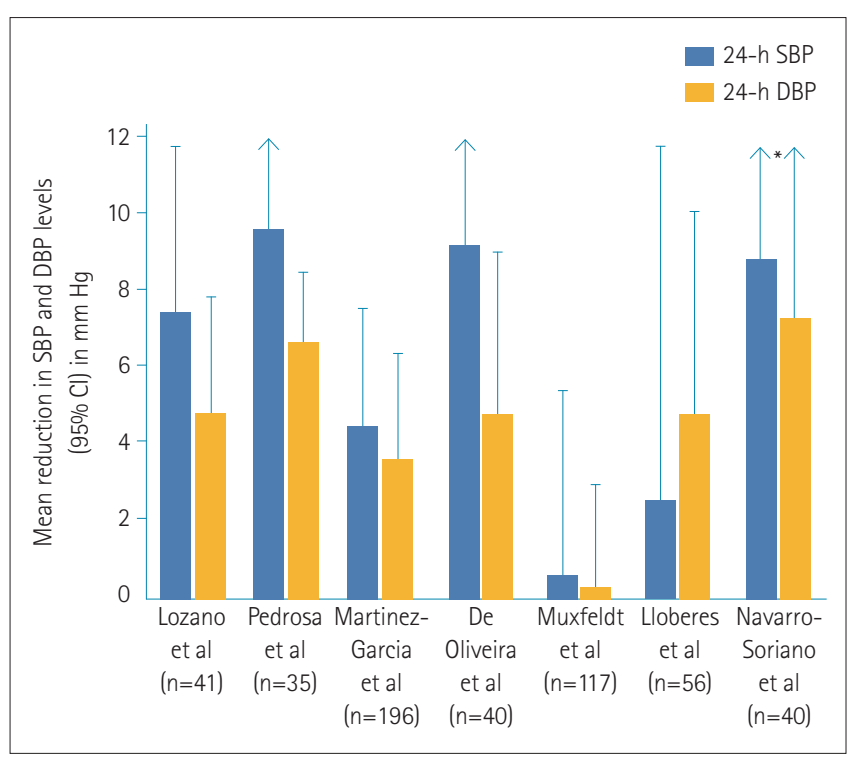

Figure 3. Antihypertensive effects of continuous positive airway pressure therapy in patients with resistant hypertension. When treated in patients with resistant hypertension, it shows more blood pressure lowering effect than in patients with simple hypertension. SBP: systolic blood pressure, DBP: diastolic blood pressure. Adapted from Oscullo et al. J Clin Med 2019;8:1872, under the terms of the Creative Commons Attribution (CC BY) license [14].

lowering the general sympathetic nervous system activation. Even if the blood pressure-lowering effect of sleep apnea treatment is not as great as expected, considering the long-term prognosis, its preventive effect of cardiovascular and cerebrovascular diseases cannot be ignored. Sleep apnea treatment should be considered because it is also involved in many other unfavorable mechanisms such as inflammatory reactions and vascular endothelial dysfunction.

\section{PULMONARY HYPERTENSION AND SLEEP APNEA}

Since sleep-disordered breathing was considered as one of the secondary causes of pulmonary hypertension at the second Pulmonary Arterial Hypertension Conference held in 1998, it has been classified as 'Class 3-pulmonary hypertension because of lung diseases and/or hypoxia' [26,27]. It has been recognized as a risk factor of pulmonary hypertension, and its prevalence is reported as $20-50 \%[28,29]$. The mechanism by which sleep apnea causes pulmonary hypertension is relatively well known, and several possible mechanisms are involved. When sleep apnea occurs, hypoxia and hypercarbonemia developed, and hypoxia causes peripheral pulmonary vasoconstriction, causing pressure of the right ventricle to rise. At the same time, when the chest cavity expands due to upper respiratory stricture, the intrathoracic pressure drops sharply and the right ventricle pressure markedly decreases. Under these conditions, blood of the superior and inferior vena cava is pushed into right heart at once, and the pressure and volume of right ventricle sharply increase, and interventricular septum is bulging toward left ventricle. As a result, excessive expansion of the right ventricle occurs and the left ventricle is com- 
pressed, and the left ventricle cannot fully diastole, resulting in a decrease cardiac output. Although this phenomenon occurs temporarily, continuous and repeated sleep apnea for a very long period of time cause gradual vascular endothelial dysfunction and remodeling, and at the same time, left ventricular hypertrophy and diastolic dysfunction will occur. Pulmonary hypertension is caused by such a pathway, so it is better to understand sleep apnea as a disease that corresponds to group 3 pulmonary hypertension to some extent, but also to 'Class 2-pulmonary hypertension because of left heart disease'.

Regarding treatment, the effect of CPAP therapy for sleep apnea has been reported in several studies and has been reported to effectively lower pulmonary arterial pressure. Representatively, according to Imran et al. [30], a meta-analysis was conducted to investigate how continuous positive pressure treatment affects pulmonary pressure in patients without heart disease or lung disease. A total of 222 subjects, average 52.5 years old patients with pulmonary hypertension (mean pulmonary arterial pressure $=39.3 \pm$ $6.3 \mathrm{~mm} \mathrm{Hg}$ ) with severe sleep apnea reported that the pulmonary arterial pressure dropped by $13.3 \mathrm{~mm} \mathrm{Hg}$ in the continuous positive pressure treatment group. CPAP therapy was found to be effective in sleep apnea patients with pulmonary hypertension. Therefore, if sleep apnea is suspected in patients with pulmonary hypertension, polysomnography is recommended.

\section{CONCLUSION}

In recent years, the lifespan of humans is increasing, and as a result, the prevalence of chronic diseases such as hypertension and diabetes is increasing. Sleep apnea has bidirectional relationship with hypertension. In patients with hypertension, the prevalence of sleep apnea is significantly higher than that in patients without hypertension. Conversely, sleep apnea increases the incidence of high blood pressure. It was found that this also applies to the relationship with pulmonary arterial hypertension. If not, it is necessary to check if sleep apnea is accompanied. Therefore, it is necessary to carefully check whether hypertension occurs in patients with sleep apnea. On the contrary, if hypertensive patients are not treated well, it is necessary to check whether sleep apnea is accompanied. Although not mentioned above, many studies have concluded that sleep apnea without treatment increases mortality. In conclusion, sleep apnea, like hypertension and diabetes, should be considered as a risk factor that may develop cardiovascular disease and physicians must be alert to this.

\section{Acknowledgments}

None

\section{Conflicts of Interest}

The authors have no potential conflicts of interest to disclose.

\section{Author Contributions}

Writing_original draft: Jin Oh Na. Writing_review \& editing:
Woohyeun Kim, Dong Oh Kang, Jah Yeon Choi.

\section{ORCID iDs}

Jin $\mathrm{Oh} \mathrm{Na}$ (D)

https://orcid.org/0000-0002-5096-4428

Woohyeun Kim

https://orcid.org/0000-0002-6939-5958

Dong Oh Kang (D)

https://orcid.org/0000-0002-5187-8310

Jah Yeon Choi (D)

https://orcid.org/0000-0002-6793-4137

\section{REFERENCES}

1. Rana D, Torrilus C, Ahmad W, Okam NA, Fatima T, Jahan N. Obstructive sleep apnea and cardiovascular morbidities: a review article. Cureus 2020; 12:e10424.

2. Marin JM, Carrizo SJ, Vicente E, Agusti AG. Long-term cardiovascular outcomes in men with obstructive sleep apnoea-hypopnoea with or without treatment with continuous positive airway pressure: an observational study. Lancet 2005;365:1046-1053.

3. Punjabi NM, Caffo BS, Goodwin JL, Gottlieb DJ, Newman AB, O'Connor GT, et al. Sleep-disordered breathing and mortality: a prospective cohort study. PLoS Med 2009;6:e1000132.

4. Javaheri S, Barbe F, Campos-Rodriguez F, Dempsey JA, Khayat R, Javaheri $\mathrm{S}$, et al. Sleep apnea: types, mechanisms, and clinical cardiovascular consequences. J Am Coll Cardiol 2017;69:841-858.

5. Alvarez-Sabín J, Romero O, Delgado P, Quintana M, Santamarina E, Ferré A, et al. Obstructive sleep apnea and silent cerebral infarction in hypertensive individuals. J Sleep Res 2018;27:232-239.

6. Porto F, Sakamoto YS, Salles C. Association between obstructive sleep apnea and myocardial infarction: a systematic review. Arq Bras Cardiol 2017; 108:361-369.

7. Young T, Finn L, Peppard PE, Szklo-Coxe M, Austin D, Nieto FJ, et al. Sleep disordered breathing and mortality: eighteen-year follow-up of the Wisconsin sleep cohort. Sleep 2008;31:1071-1078.

8. Javaheri S, Javaheri S, Javaheri A. Sleep apnea, heart failure, and pulmonary hypertension. Curr Heart Fail Rep 2013;10:315-320.

9. Arias MA, García-Río F, Alonso-Fernández A, Martínez I, Villamor J. Pulmonary hypertension in obstructive sleep apnoea: effects of continuous positive airway pressure: a randomized, controlled cross-over study. Eur Heart J 2006;27:1106-1113.

10. McLaughlin VV, Archer SL, Badesch DB, Barst RJ, Farber HW, Lindner JR, et al. ACCF/AHA 2009 expert consensus document on pulmonary hypertension a report of the American College of Cardiology Foundation Task Force on Expert Consensus Documents and the American Heart Association developed in collaboration with the American College of Chest Physicians; American Thoracic Society, Inc.; and the Pulmonary Hypertension Association. J Am Coll Cardiol 2009;53:1573-1619.

11. Bradley TD, Floras JS. Obstructive sleep apnoea and its cardiovascular consequences. Lancet 2009;373:82-93.

12. Somers VK, Dyken ME, Clary MP, Abboud FM. Sympathetic neural mechanisms in obstructive sleep apnea. J Clin Invest 1995;96:1897-1904.

13. Kasai T, Bradley TD. Obstructive sleep apnea and heart failure: pathophysiologic and therapeutic implications. J Am Coll Cardiol 2011;57:119-127.

14. Oscullo G, Torres G, Campos-Rodriguez F, Posadas T, Reina-González A, Sapiña-Beltrán E, et al. Resistant/refractory hypertension and sleep apnoea: current knowledge and future challenges. J Clin Med 2019;8:1872.

15. Korea Centers for Disease Control and Prevention. 2017 Korea National Health and Nutrition Examination Survey (KNHANES VII-2). Sejong: Ministry of Health and Welfare; 2018.

16. Kim J, In K, Kim J, You S, Kang K, Shim J, et al. Prevalence of sleep-disordered breathing in middle-aged Korean men and women. Am J Respir Crit Care Med 2004;170:1108-1113.

17. Silverberg DS, Oksenberg A, Iaina A. Sleep-related breathing disorders as a 
major cause of essential hypertension: fact or fiction? Curr Opin Nephrol Hypertens 1998;7:353-357.

18. Sjöström C, Lindberg E, Elmasry A, Hägg A, Svärdsudd K, Janson C. Prevalence of sleep apnoea and snoring in hypertensive men: a population based study. Thorax 2002;57:602-607.

19. Marin JM, Agusti A, Villar I, Forner M, Nieto D, Carrizo SJ, et al. Association between treated and untreated obstructive sleep apnea and risk of hypertension. JAMA 2012;307:2169-2176.

20. Barbé F, Durán-Cantolla J, Sánchez-de-la-Torre M, Martínez-Alonso M, Carmona C, Barceló A, et al. Effect of continuous positive airway pressure on the incidence of hypertension and cardiovascular events in nonsleepy patients with obstructive sleep apnea: a randomized controlled trial. JAMA 2012;307:2161-2168.

21. Logan AG, Perlikowski SM, Mente A, Tisler A, Tkacova R, Niroumand M, et al. High prevalence of unrecognized sleep apnoea in drug-resistant hypertension. J Hypertens 2001;19:2271-2277.

22. Ruttanaumpawan P, Nopmaneejumruslers C, Logan AG, Lazarescu A, Qian I, Bradley TD. Association between refractory hypertension and obstructive sleep apnea. J Hypertens 2009;27:1439-1445.

23. Martínez-García MA, Navarro-Soriano C, Torres G, Barbé F, CaballeroEraso C, Lloberes P, et al. Beyond resistant hypertension. Hypertension 2018;72:618-624.

24. Pedrosa RP, Drager LF, Gonzaga CC, Sousa MG, de Paula LK, Amaro AC, et al. Obstructive sleep apnea: the most common secondary cause of hypertension associated with resistant hypertension. Hypertension 2011;58:811817.

25. Bazzano LA, Khan Z, Reynolds K, He J. Effect of nocturnal nasal continuous positive airway pressure on blood pressure in obstructive sleep apnea. Hypertension 2007;50:417-423.

26. Simonneau G, Gatzoulis MA, Adatia I, Celermajer D, Denton C, Ghofrani A, et al. Updated clinical classification of pulmonary hypertension. J Am Coll Cardiol 2013;62:D34-D41.

27. Nathan SD, Barbera JA, Gaine SP, Harari S, Martinez FJ, Olschewski H, et al. Pulmonary hypertension in chronic lung disease and hypoxia. Eur Respir J 2019;53:1801914.

28. Atwood CW Jr, McCrory D, Garcia JG, Abman SH, Ahearn GS; American College of Chest Physicians. Pulmonary artery hypertension and sleep-disordered breathing: ACCP evidence-based clinical practice guidelines. Chest 2004;126:72S-77S.

29. Floras JS. Sleep apnea and cardiovascular disease: an enigmatic risk factor. Circ Res 2018;122:1741-1764.

30. Imran TF, Ghazipura M, Liu S, Hossain T, Ashtyani H, Kim B, et al. Effect of continuous positive airway pressure treatment on pulmonary artery pressure in patients with isolated obstructive sleep apnea: a meta-analysis. Heart Fail Rev 2016;21:591-598. 